moniser, sur le plan juridique, le système traditionnel de protection des espèces végétales cultivées et le système des dépôts de brevet associé au développement des technologies nouvelles. Les autres ont trait, plus spécifiquement, au Sud et à la relation Nord-Sud. D'une part est affirmée la nécessité de protéger la diversité des espèces végétales naturelles. D'autre part, est soulignée l'importance du fossé Nord-Sud en matière de systèmes technologiques, qui engendre une double inquiétude de la part des pays du Sud : les biotechnologies sont développées par des firmes privées dans et pour les pays industrialisés et la crainte peut exister d'une dépossession juridique des pays du Sud au profit de ceux qui ont le monopole des nouvelles recherches et de la commercialisation des nouveaux produits; en outre le risque existe d'une déstabilisation économique liée à l'apparition de produits de substitution, éventuellement élaborés à partir de produits du Sud et ultérieurement réexportés.

En ce domaine, comme dans celui de la santé, les biotechnologies peuvent être une chance pour le développement, mais les recommandations sont d'autant plus nécessaires que la réalité actuelle, malgré les diverses formes de coopération (bilatérales, interrégionales ou autres), est, à quelques exceptions près, préoccupante.

On ne peut qu'être sensible, à l'issue de ces journées et de ces débats, à l'importance inédite et fondamentale des questions aujourd'hui soulevées par le développement des biotechnologies. Importance inédite, en ce sens que jamais n'avaient pu être formulées jusqu'alors des hypothèses auxquelles les progrès de la science et la technologie donnent un tel crédit. Mais importance fondamentale car l'histoire et l'anthropologie illustrent surabondamment la nature première et universelle des thèmes induits par les possibilités nouvelles : les interrogations sur l'identité individuelle, sur la relation entre le même et l'autre, sur la nature de la filiation, de la transmission et de l'hérédité composent non seulement la trame des angoisses ou des fantasmes individuels à l'écoute desquels essaient de se mettre les psycha-

mythologies, des cosmologies et des anthropologies élaborées par les diverses cultures du monde. Il y a donc urgence anthropologique et historique à prendre la mesure exacte des nouvelles possibilités qui s'offrent à l'humanité.

Et comme toujours lorsque se formule un problème de société, deux voies se proposent. Que ce soit dans le domaine de l'information ou dans celui de l'action, dans le domaine de la recherche ou celui de la loi, il faut choisir entre la mise à l'écart et la mise en commun : que chaque individu ait droit à l'information et que la génétique soit l'affaire de toute l'humanité, c'est une vérité qui ne s'apparentera pas simplement à un vou pieux dès lors qu'elle sera au principe de la recherche des scientifiques et de l'action des décideurs

\title{
Nouvelles orientations pour la thérapie génique
}

\section{Axel Kahn, Pascale Briand}

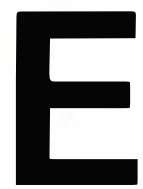

tant acquis qu'il n'y a ni indication ni légitimité à la modification génétique héréditaire de l'embryon précoce (Tableaul), au moins en l'état actuel de nos connaissances, les efforts des « thérapeutes géniques » se concentrent déjà depuis plusieurs années sur la thérapie génique somatique, c'est-à-dire sur l'apport d'un gène fonctionnel à des cellules n'intervenant pas dans la gamétogenèse et possédant un équivalent anormal du gène transféré.

\section{Les greffes}

En un sens, les greffes d'organes pratiquées pour traiter des maladies héréditaires peuvent être considérées comme une thérapie génique, le but en étant bien, au moins en partie, l'apport d'un gène fonctionnel. A dire vrai, ce sont même pour l'instant les seules thérapies géniques à avoir fait la preuve de leur efficacité : greffes de moelle osseuse pour des hémoglobinopathies graves ou des déficits immunitaires congénitaux, greffes de foie pour des affections en rapport avec des troubles héréditaires du métabolisme hépatique, voire greffes de poumons ou de blocs coeur-poumons chez des mucoviscidosiques, etc. Les progrès extrêmement rapides réalisés dans le domaine des greffes, l'apparition d'immunosuppresseurs de plus en plus efficaces et de moins en moins toxiques font des greffes une réelle perspective dans nombre de maladies héréditaires. Les exigences de compatibilité immunologique, le manque de disponibilité des organes à greffer, les risques de contamination virale des greffons, la nécessité d'un traitement immunosuppresseur et d'une surveillance prolongée des malades greffés ne permettent cependant pas de considérer cette stratégie autrement que comme un pis-aller justifié par l'absence actuelle d'autres perspectives.

\section{Autogreffes de cellules génétiquement modifiées}

Dans son principe, cette approche est extrêmement attrayante : il s'agit en effet d'utiliser les propres cellules du malade (ce qui supprime tout problème immunologique), de les modifier par introduction du gène sensé compenser l'anomalie génétique, puis de les réintroduire dans l'organisme (figure 1). Avant même de passer à l'expérimentation, des questions surgissent cependant. La première est celle de l'inocuité de l'introduction, au hasard dans le génome, d'un fragment d'ADN, le plus souvent apporté par un vecteur rétroviral(figure 2). Les risques potentiels d'activation d'un oncogène situé à proximité du site d'intégration de l'ADN exogène sont bien sûr diminués autant qu'il est possible par utilisation de vecteurs dont les enhancers (situés dans les LTR, long terminal repeats) sont inactivés. De plus, l'absence d'apparition de cancers spontanés chez les souris transgéniques ayant intégré, 


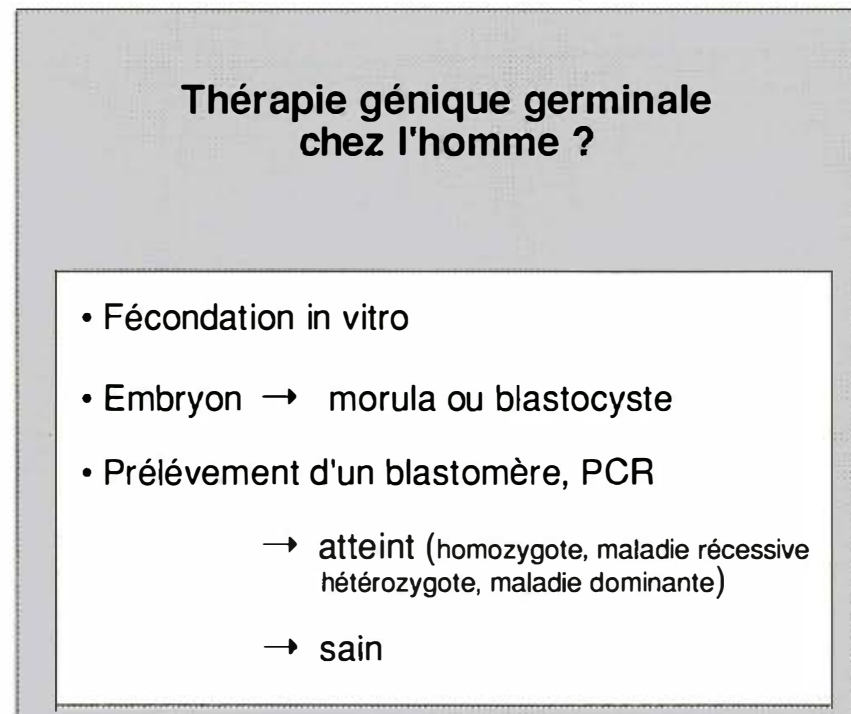

TRI, et nonTHÉRAPIE !

Tableau I. La thérapie germinale chez l'homme ne serait envisageable qu'après un diagnostic de la maladie sur des embryons issus de FN et maintenus in vitro jusqu'a un stade morula ou blastocyte. Le diagnostic se ferait par utilisation de la PCR. Puisque dans tous les cas, on se retrouverait devant un mélange d'embryons sains et malades, c'est le tri d'embryon qui s'imposerait, pas la thérapie gènique.

\begin{tabular}{|c|c|c|c|}
\hline \multirow{2}{*}{$\begin{array}{l}\text { TYPE DE MALADIE } \\
\text { HÉREDDITAIRE }\end{array}$} & \multicolumn{2}{|c|}{ THÉRAPIE GÉNIQUE } & \multirow{2}{*}{ ALLOGREFFE } \\
\hline & Autogreffe & $\begin{array}{l}\text { Vecteur } \\
\text { in vivo }\end{array}$ & \\
\hline $\begin{array}{l}1 \text { organe responsable } \\
\text { non atteint } \\
\text { (maladies de la coagulation, } \\
\text { hypercholestérolémie, } \\
\text { oxalose, } \\
\text { déficit immunitaires) }\end{array}$ & + & & +, Fait \\
\hline $\begin{array}{l}1 \text { organe responsable et } \\
\text { atteint, greffable } \\
\text { (maladies de l'hémogiobine, } \\
\text { déficit en a1 AT, tyrosinémie, } \\
\text { glycogénose) }\end{array}$ & + (Moelle) & & +. Fait \\
\hline $\begin{array}{l}\text { Organe atteint } \\
\text { non greffable, } \\
\text { ou tous les tissus } \\
\text { (Tay Sachs, myopathie, } \\
\text { mucoviscidose) }\end{array}$ & & + & \\
\hline
\end{tabular}

Tableau III. Types de thérapie génique ou de greffe d'organe adaptées à diverses situations de maladies héréditaires.

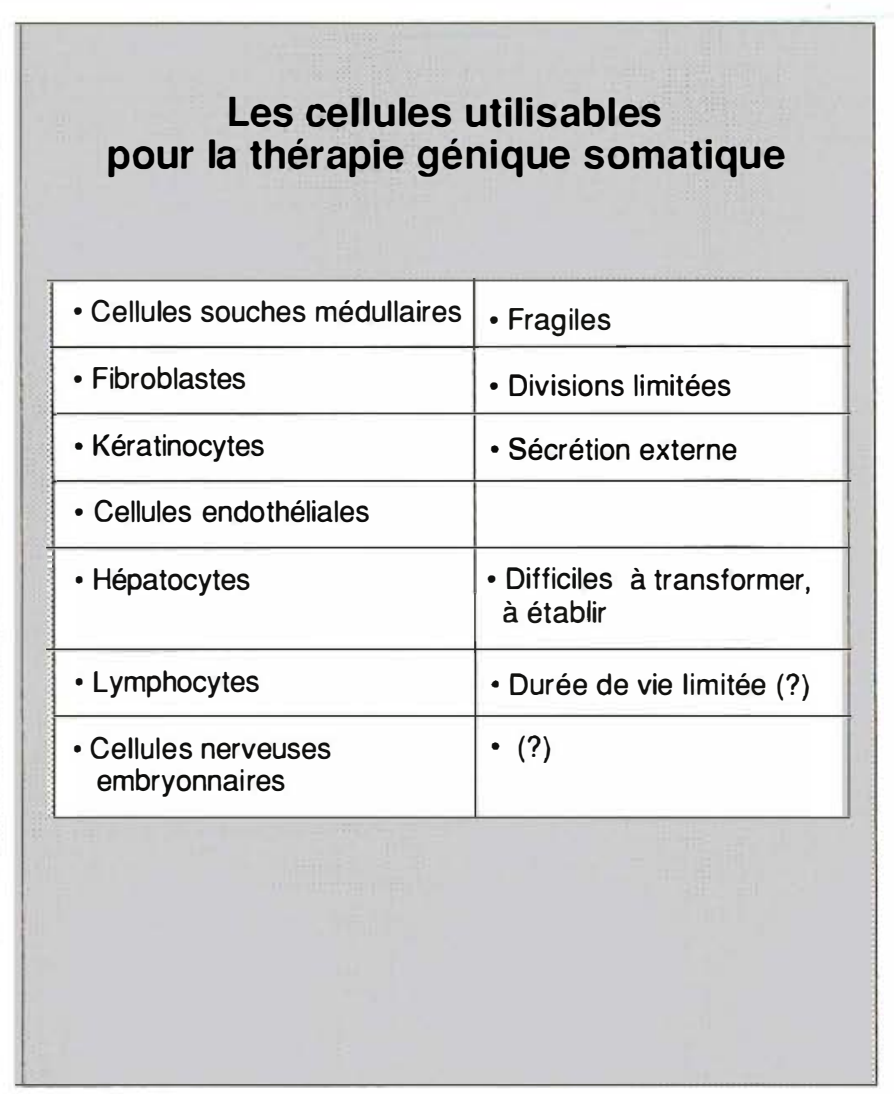

Tableau II.

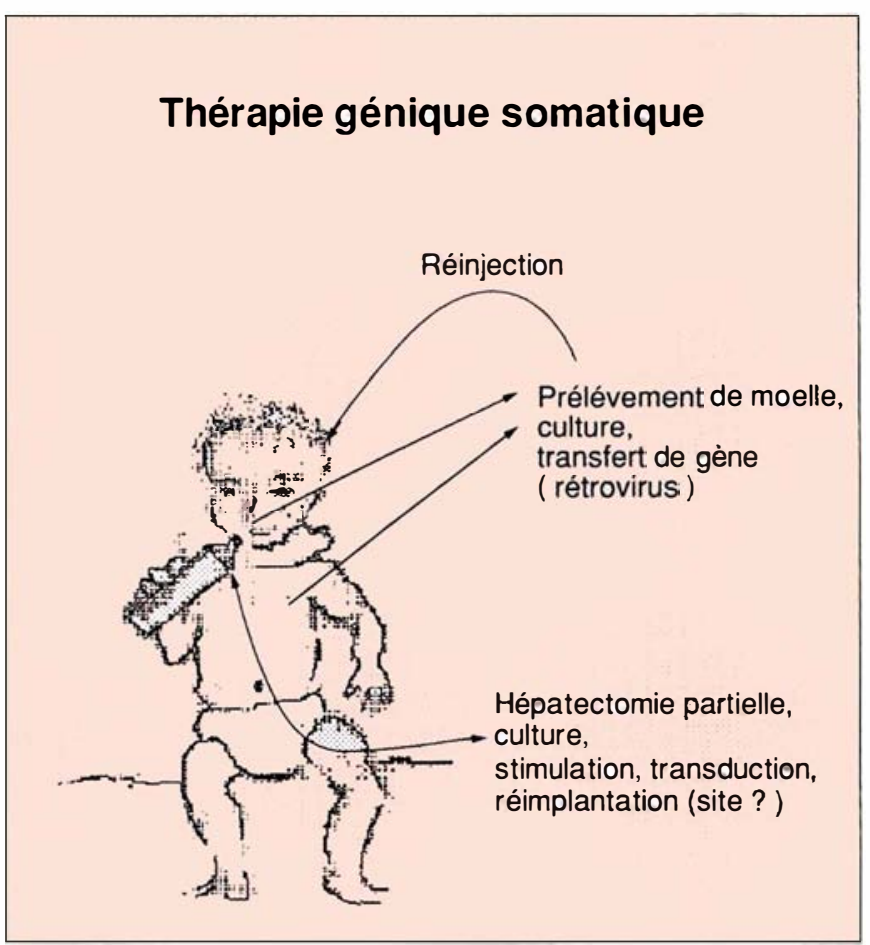

Figure 1. La thérapie génique somatique peut consister en prélévement chez l'enfant atteint de cellules malades (moelle osseuse ou hépatocytes, par exemple), en l'introduction in vitro du gène curateur, puis en la réimplantation des cellules génétiquement modifiées. 
au hasard dans leur génome, des séquences d'ADN non oncogéniques semble indiquer que le processus d'activation insertionnelle n'est pas fréquent. Le risque théorique en persiste néanmoins et, quand bien même la méthode aurait par ailleurs démontré son efficacité, resterait probablement un obstacle durable aux autorisations larges d'essais chez l'homme; les décisions seraient alors prises au cas par cas, tenant compte, comme dans tout essai thérapeutique, de l'efficacité supposée, de la gravité de l'affection à traiter et de l'évaluation du risque potentiel.

Les incertitudes persistantes à propos des conséquences éventuelles d'une insertion au hasard d'ADN exogène dans le génome cellulaire expliquent que beaucoup d'espoirs soient désormais placés dans la « recombinaison homologue» qui permet d'intégrer un fragment d'ADN a u sein de la séquence homologue de l'ADN chromosomique, voire de réellement «réparer » un gène modifié par mutation. L'approche consisterait ici à sélectionner par culture in vitro les cellules des malades sièges d'un événement de recombinaison homologue, comme cela est décrit en détail dans l'article de Valérie Lemarchandel et Xavier Montagutelli ( $\mathrm{m} / \mathrm{s} n^{\circ} 1$, vol. 6, p. 18), puis à réimplanter ces cellules. Malheureusement, les récents résultats obtenus par R.L. Brinster et al., en collaboration avec R.D. Palmiter (Philadelphie, PA, et Seattle, WA, USA) indiquent que le processus de recombinaison homologue peut s'accompagner de l'introduction de maintes mutations risquant d'inactiver le gène que l'on désirait réparer [1].

Une autre difficulté, évidente a priori et confirmée a posteriori, réside en l'exigence que les cellules génétiquement modifiées soient, notamment, des cellules souches douées d'autorenouvellement, et non des cellules déjà engagées dans une voie donnée de différenciation terminale. Dans ce dernier cas, en effet, on peut s'attendre à la disparition rapide des cellules modifiées, à la vitesse de leur différenciation irréversible en des cellules à durée de vie limitée.

Enfin, exigence supplémentaire, il faut s'assurer que le gène transféré 146 niveaux suffisants pour avoir une réelle action curative. A la lumière de tous ces prérequis, il semblait probable, jusqu'à il y a une année, que les maladies curables par l'intermédiaire de cellules médullaires génétiquement modifiées seraient les premières à faire l'objet de tentatives thérapeutiques. Il ne s'agissait pas tant des maladies moléculaires de l'hémoglobine exigeant que les cellules modifiées synthétisent la chaîne de globine codée par le gène transféré en quantité énorme, ajustée à la synthèse endogène de la chaîne non intéressée par la maladie, que de carences immunitaires rares dues à des déficits enzymatiques intéressant le métabolisme des nucléotides puriques (adénosine désaminase et purine nucléosides phosphorylase). Les conditions semblaient particulièrement favorables: la moelle est facile à prélever, le succès des greffes de moelle indique que des cellules souches sont bien présentes dans ces prélèvements, et beaucoup d'éléments indiquent qu'une correction partielle des déficits enzymatiques devrait être suffisante pour supprimer la carence immunitaire. En réalité les difficultés expérimentales rencontrées chez l'animal furent considérables, liées soit à l'altération des cellules souches médullaires au cours de la culture in vitro nécessaire à l'infection par les rétrovirus recombinés, soit à la moindre infectabilité des cellules souches comparées aux cellules déjà engagées dans les différentes voies hématopoïétiques. De plus, des réarrangements associés à l'intégration de l'ADN exogène sont apparus fréquents. Jusqu'à très récemment, par conséquent, on n'était pas parvenu à maintenir durant une longue période un haut niveau d'expression de l'ADN exogène transféré à l'animal d'expérience via l'infection rétrovirale de cellules médullaires [2]. Une série de progrès récents permettent néanmoins d'être plus optimiste. Il s'agit de la simplification maximale des vecteurs, de l'utilisation de promoteurs internes de préférence au promoteur rétroviral des LTR et de la limitation au strict minimum des manipulations in vitro des cellules médullaires.

De plus, l'infectabilité des cellules souches par les rétrovirus peut être considérablement stimulée en provoquant leur entrée dans le cycle cellulaire grâce à l'action de cytokines, notamment les interleukines 3 et 6 [ 3 , $4,5]$. Grâce à ces perfectionnements, des expressions très prolongées de gènes exogènes introduits dans des cellules médullaires greffées ont été obtenues chez la souris.

Les candidats «thérapeutes géniques» se sont aussi tournés vers d'autres cellules receveuses éventuelles qui accepteraient de se laisser modifier et d'engendrer in vivo des lignées permanentes (Tableau III). Les fibroblastes ne semblent pas de bons candidats, car leur capacité de division est limitée $\left(\mathrm{m} / \mathrm{s} n^{\circ} 7\right.$, vol. 3, p. 425). Les kératinocytes ont quelques propriétés intéressantes... mais auraient plus tendance à sécréter les produits des gènes transférés à l'extérieur, vers la peau, que dans la circulation sanguine. Les cellules endothéliales ont des propriétés très attrayantes: elles sont au contact direct du flot sanguin, semblent douées d'autorenouvellement et peuvent être apportées par l'intermédiaire de greffons vasculaires $(\mathrm{m} / \mathrm{s}$ $n^{\circ} 7$, vol.5, p.511). Fibroblastes, kératinocytes et cellules endothéliales génétiquement recombinés peuvent non seulement apporter à l'organisme des protéines dont elles assurent habituellement la synthèse, mais aussi des molécules normalement produites par d'autres cellules. Ainsi la production de facteur IX normalement synthétisé par les hépatocytes peut être assurée par des kératinocytes ou des cellules endothéliales qui vont le déverser en quantité appréciable dans la circulation [6].

Les lymphocytes entrent aussi dans cette catégorie de cellules, potentielles usines de production d'un facteur manquant. Ils doivent être mis à part, principalement parce qu'ils sont le premier type de cellules à avoir été utilisé pour une expérience de transfert de gène chez l'homme et que nous possédons par conséquent des premières indications sur leur comportement après infection par un rétrovirus transportant un gène étranger, en l'occurrence un marqueur de résistance à un antibiotique actif sur les cellules de mammifères ( $m / s n^{\circ} 9$, vol. 5, p. 698). Il s'agissait 

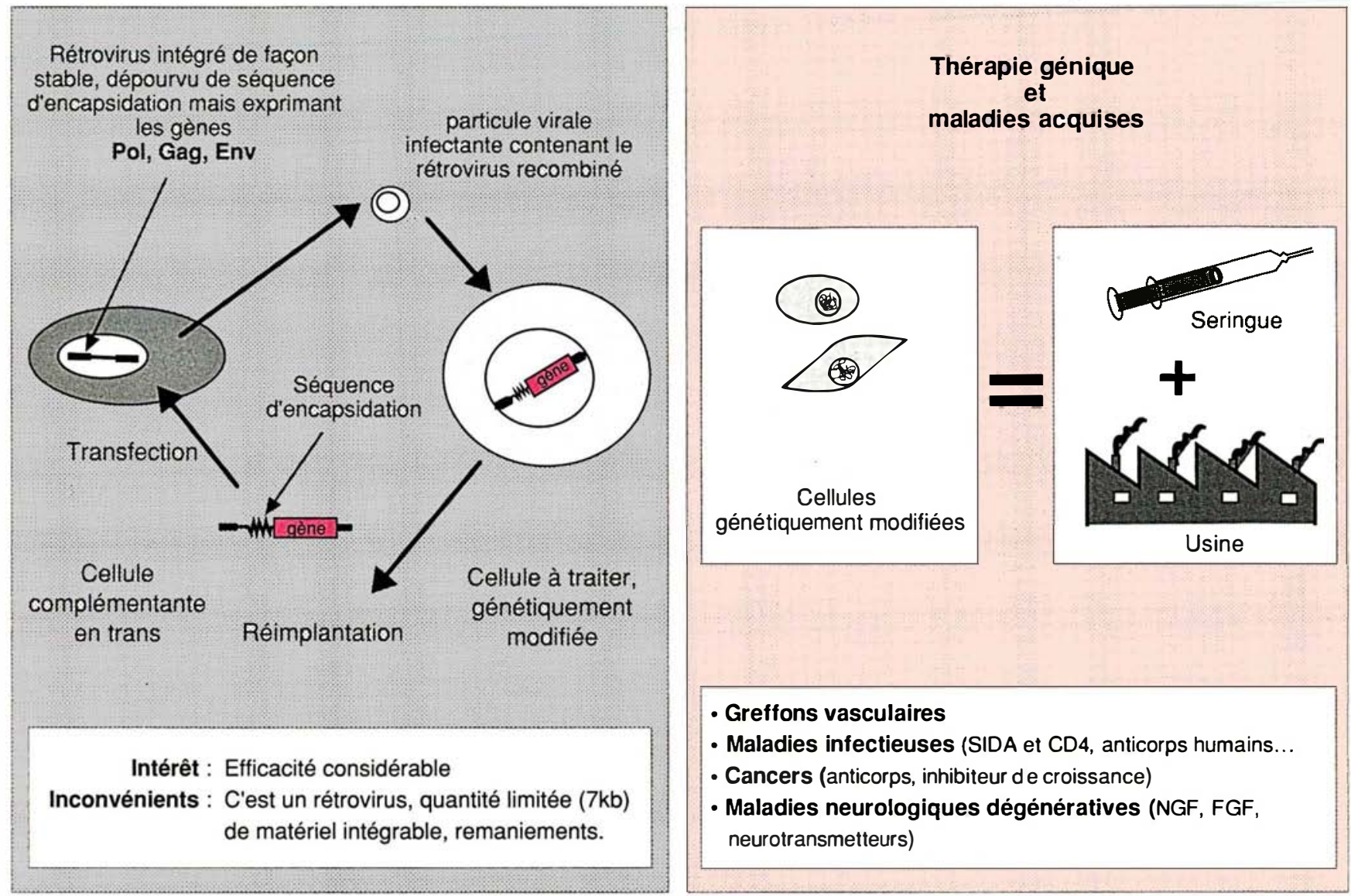

Figure 2. Utilisation de rétrovirus à des fins de thérapie Figure 4. génique somatique

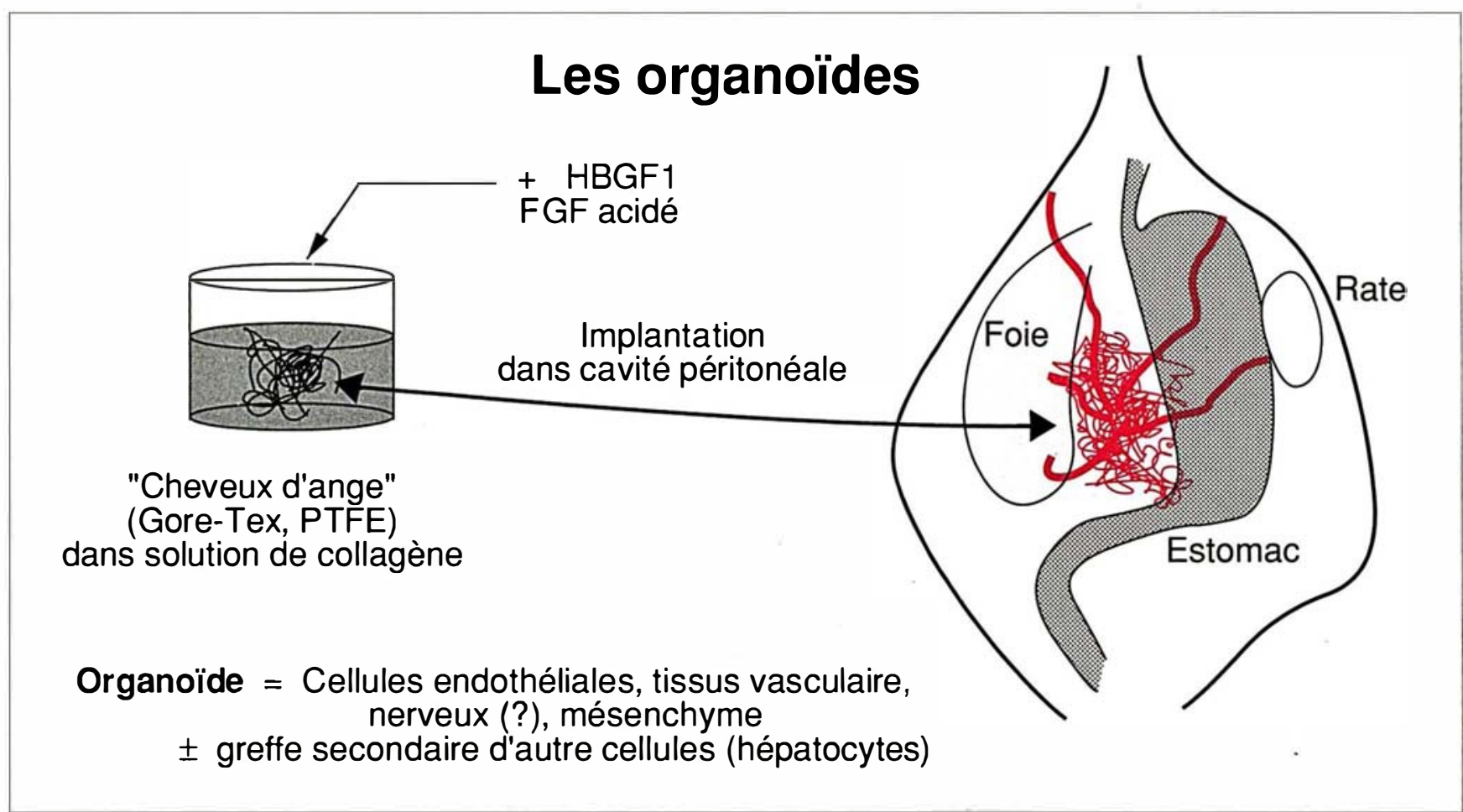

Figure 3. 
de suivre le devenir de TIL (tumor infiltrating lymphocytes) chez des malades souffrant de mélanomes métastasés. Ces lymphocytes peuvent en effet, après activation par l'interleukine-2, avoir un effet antitumoral, les échecs pouvant être dus à l'incapacité des TIL réinjectés de se concentrer au niveau des tumeurs. Quoiqu'il soit très improbable avec ces cellules de parvenir à une colonisation définitive in vivo, les expériences menées chez les malades atteints de mélanome ont montré qu'elles pouvaient survivre, dans de bonnes conditions, plusieurs mois. Faciles à prélever, faciles à injecter, les lymphocytes sont donc peut-être une solution intéressante, soit que l'on envisage de traiter une maladie génétique (par exemple un déficit immunitaire) en répétant les injections de cellules génétiquement modifiées, soit que la thérapie génique soit utilisée, comme nous le verrons plus loin, pour traiter une maladie non génétique, éventuellement transitoire.

Les hépatocytes, malgré la difficulté de leur manipulation in vitro et leur faible degré d'infectabilité par les rétrovirus, ont fait l'objet de nombreux essais de transfert parce que le traitement de maladies héréditaires variées du métabolisme nécessite une correction de l'anomalie dans ce type cellulaire. La faisabilité des réimplantations d'hépatocytes, recombinés génétiquement ou non, a été démontrée. De telles mini-greffes ont par exemple permis de faire apparaître de la bilirubine conjuguée dans la bile de rats Gunn déficients en UDP. glucuronyl transférase, ainsi que de l'albumine dans le sang de rats analbuminémiques $\left(m / s n^{\circ} 5\right.$, vol. 3 , p. 303).

En revanche, des hépatocytes infectés par des rétrovirus contenant le gène du récepteur des $\mathrm{LDL}$ et réinjectés dans la cavité péritonéale de lapins Watanabe, déficients en ce récepteur, n'ont pas permis d'obtenir un résultat appréciable et durable sur l'hypercholestérolémie de ces animaux [7].

Par conséquent, si l'on peut considérer que la réintroduction des hépatocytes ne pose pas de problèmes particuliers, les niveaux d'expressions et la persistance d'une telle expression après transfert restent de faible importance. De plus, une stratégie classique de thérapie génique effectuée sur les hépatocytes nécessiterait leur prélèvement sur le malade par hépatectomie partielle. Sans être impossible, la difficulté de chaque étape rend probablement lointaine l'application d'une telle approche.

\section{Les organoïdes}

Très récemment, plusieurs équipes de la côte Est des Etats-Unis ont décrit une technique remarquable qui pourrait faciliter considérablement la thérapie génique somatique, notamment celle utilisant les hépatocytes: il s'agit de la création d'orga noïdes [8]. médecine/science a longuement traité de l'angiogenèse, préalable indispensable à la croissance des tissus, normaux et tumoraux $\left(m / s n^{\circ} 5\right.$, vol. 4, p. 318 et $n^{\circ} 7$, vol. 5 , p. 516). Les facteurs angiogéniques sont nombreux; beaucoup d'entre eux ont la propriété de se fixer à l'héparine ou à des glycosaminoglycanes de la matrice extracellulaire. Ils forment ainsi la famille des HBGF (heparin-binding growth factors). HBGF-l est identique au FGF acide (fibroblast growth factor) alors que HBGF-2 est le FGF basique.

La formation d'organoïdes est simple (figure 3). Un support non résorbable et bien toléré par l'organisme, le polytetrafluoréthylène (PTFE, Gore-Tex) est utilisé sous la forme de fins filaments non tissés («cheveux d'anges »); il est incubé dans une solution de collagène de manière à recouvrir les fibres textiles de cette protéine de la matrice extracellulaire, puis dans une solution d'HBGF1/FGF acide. Ce dernier facteur de croissance est si puissant que quelques centaines de nanogrammes suffisent. La mixture est alors implantée dans la cavité péritonéale de rats. En quelques semaines, de complexes structures vasculaires (artériolaires, capillaires, lymphatiques et veineuses) se développent au sein d'un mésenchyme abondant qui semble aussi contenir, fait plus surprenant, des formations nerveuses. Selon le site d'implantation, l'organoïde, qui semble parfaitement viable aussi longtemps que dure l'expérience (plus de six mois) échange de nombreuses connections vasculaires avec les tissus environnants, foie, rate, péritoine, anses intestinales, etc. Des hépatocytes en suspensions injectés dans un organoïde constitué s'y établissent et restent fonctionnels durant toute la période d'examen, c'est-à-dire six mois. Ils sont capables de provoquer une diminution de plus de moitié de la bilirubine sérique de rats Gunn.

\section{Thérapie génique de maladies génétiques et non génétiques}

Les indications de la thérapie génique somatique par autogreffe de cellules génétiquement modifiées incluent des maladies génétiques et des affections acquises. Les maladies génétiques les plus accessibles à ce traitement (Tableau III) sont celles qui sont secondaires au déficit d'une protéine circulante (maladies de la coagulation, qu'ils s'agissent des hémophilies ou des maladies thrombotiques congénitales [9], déficits hormonaux congénitaux, etc.) et celles dans lesquelles un organe, le foie par exemple est la cause de la maladie, mais non sa victime : oxalose; maladie de Crigler-Najjar, équivalent humain du déficit du rat Gunn; hypercholestérolémie familiale ; anomalies du cycle de l'urée ou de la néoglucogenèse, etc. [10]. Un déficit hétérozygote en $\alpha$-l antitrypsine pourrait être une indication de thérapie génique selon ces méthodes, afin d'éviter la destruction du tissu pulmonaire et l'emphysème. En revanche, parce qu'il s'accompagne d'un risque important de cirrhose et d'hépatocarcinome par surcharge en la protéine mutée, un déficit homozygote de phénotype $Z$ resterait, pour l'instant, une indication de la greffe hépatique $\left(\mathrm{m} / \mathrm{s} n^{\circ} 3\right.$, vol. 3, p. $181 \mathrm{et}$ $n^{\circ} 3$, vol. 5, p. 182).

Les maladies non génétiques que l'on peut envisager de traiter par thérapie génique sont, en théorie, nombreuses. Les cellules génétiquement modifiées sont ici considérées comme des mini-usines de production et de distribution d'une substance d'intérêt biologique: facteurs thrombolytiques libérés par des cellules endothéliales tapissant un greffon vasculaire; anticorps monoclonal «humanisé» (c'est-à-dire possédant des régions constantes humaines) dans une immunothéra- 
pie passive anti-infectieuse ou antitumorale; dérivés solubles de CD4 chez les malades atteints de SIDA, facteurs neurotrophiques libérés par des cellules neuronales embryonnaires implantées dans le cerveau de sujets atteints de maladies neurodégénératives [11], etc.

\section{Injection de particules virales}

L'introduction directe d'un gène correcteur dans l'organisme pourrait permettre d'ajouter à la liste des candidats à une thérapie génique les malades atteints d'anomalies héréditaires nécessitant d'être corrigées dans une très large proportion de leurs cellules. C'est le cas, par exemple, des mucoviscidosiques ou des myopathes. Cette approche permettrait en outre un traitement très précoce parfois rendu nécessaire par l'apparition de symptômes dès la période néonatale. La thérapie génique directe se heurte malheureusement à deux difficultés majeures. La première est celle déjà soulevée des risques d'activation d'oncogènes par l'insertion des séquences introduites. La recombinaison homologue, qui à ce jour n'est pas contrôlée mais seulement repérable ex vivo, ne peut ici être utilisée pour écarter ce danger. La recherche de vecteurs viraux performants mais présentant le minimum de risques pathogènes est donc particulièrement importante pour rendre applicable cette stratégie. A côté des rétrovirus délétés de leurs séquences régulatrices, les adénovirus peuvent être considérés comme de bons candidats [12]. Il n'est cependant pas exclu qu'ils puissent occasionner des symptomatologies plus sévères que la classique rhinopharingite. La seconde difficulté majeure de cette approche est que les cellules germinales ne sont pas à l'abri du traitement. Même l'absence de tropisme d'un virus donné vis-à-vis des spermatozoïdes et des ovules n'écarterait pas tout danger car l'on sait bien que le tropisme est plus lié à la possibilité d'expression que de pénétration du virus dans une cellule. Or le danger est bien dans la pénétration et l'intégration dans le germen de cet ADN viral recombinant qui pourrait modifier l'espèce. Par conséquent, s'il $s$ 'avérait nécessaire, dans quelques cas $m / s n^{\circ} 2$ vol. 6, féurier 90 particuliers, d'opter pour cette stratégie, il faudrait peut-être envisager d'adjoindre un traitement contraceptif définitif. Il faut mentionner qu'une telle contraception est en fait déjà associée à des traitements de maladies graves (chimiothérapies anticancéreuses en particulier, cancers du sein hormono-dépendants, certains cancers du testicule).

A côté des vecteurs viraux, d'autres véhicules ont été envisagés pour introduire de l'ADN in vivo, mais les résultats sont restés décevants. Citons pour mémoire les liposomes, initialement développés pour tenter des thérapies enzymatiques. Ces véhicules sont potentiellement intéressants car ils devraient permettre de cibler l'ADN vers un type cellulaire donné et par conséquent d'éliminer tout risque d'atteinte des cellules germinales. Par exemple, les liposomes portant à leur surface des glycoprotéines dont les acides sialiques ont été hydrolysés peuvent être reconnus par les récepteurs hépatiques des asialoglycoprotéines et permettre un ciblage dans les hépatocytes [13]. Il n'existe cependant pas assez de résultats expérimentaux pour affirmer qu'ils constituent un outil adapté, efficace et sans conséquences délétères propres.

En conclusion, en partie à cause des difficultés rencontrées dans la mise au point des thérapies géniques de déficits héréditaires initialement supposés être de bons candidats à une telle approche thérapeutique, les chercheurs se sont tournés vers la recherche de transporteurs de gènes, de cellules cibles, et de modes de réintroduction dans l'organisme des cellules corrigées, entièrement nouveaux, tels les cellules endothéliales et les organoïdes.

Cette évolution des méthodes envisagées s'est accompagnée d'une dérive progressive des indications de la thérapie génique: alors que le traitement par ces moyens des maladies héréditaires les plus fréquentes et les plus graves (mucoviscidose, maladies de l'hémoglobine, myopathies, maladies lysosomales avec encéphalopathie, etc.) reste probablement encore très lointain, il est récemment apparu que nombre de maladies acquises pourraient bénéficier dans un avenir proche de l'introduction de cellules génétiquement modifiées
RÉFÉRENCES

1. Brinster RL, Braun RE, Lo D, Avarbock MR, Oram F, Palmiter R. Targeted correction of a major histocompatibility class II $\mathrm{E} \alpha$ gene by DNA microinjected into mouse eggs. Proc Natl Acad Sci USA 1989 ; 86 : 7087. 91 .

2. Culliton BJ. Designing cells to deliver drugs. Science 1989; 246 : 746 .

3. Bodine DM, Karlsson S, Nienhuis AW. Combination of interleukins 3 and 6 preserves stem cell function in culture and enhances retrovirus-mediated gene transfer into hematopoietic stem cells. Proc Natl Acad Sci USA 1989; 86 : 8897-901.

4. Correll PM, Fink JK, Brady RO, Perry LK, Karlsson S. Production of human glucocerebrosidase in mice after retroviral gene transfer into multipotential hematopoietic progenitor cells. Proc Natl Acad Sci USA 1989; 86 : 8912-6.

5. Lim B, Apperley JF, Orkin SH, Williams DA. Long term expression of human adenosine deaminase in mice transplanted with retrovirus-infected hematopoietic stem cells. Proc Natl Acad Sci USA 1989 ; 86 : 8892 6.

6. Garver RI, Chiytil A, Courtney M, Crystal RG. Clonal gene therapy: transplanted mouse fibroblast clones express human $\alpha-1$ antitrypsin gene in vivo. Science, 1989; 237 : 762-4.

7. Wilson JM, Johnston DE, Jefferson DM, Mulligan RC. Correction of the genetic defect in hepatocytes from the Watanabe heritable hyperlipemic rabbit. Proc Natl Acad Sci USA $1988 ; 85: 4421-5$.

8. Thompson JA, Haudenschild CC, Anderson KD, Dipieuro JM, Anderson F, Maciag T. Heparin binding growth factor 1 induces the formation of organoid neovascular structures in vivo. Proc Natl Acad Sci USA 1989; 86 : 7928-32.

9. Boyer-Neumann C, Wolf M, Larrieu MJ Les maladies thrombo-emboliques constitutionnelles. médecine/sciences $1987 ; 4: 216-22$.

10. Bernard O, Fischer A, Grünfeld JP. La transplantation d'organe comme traitement des maladies métaboliques héréditaires. médecine/sciences 1988; 7 : 406-12.

11. Wolff JA, Fisher LJ, Xu L et al. Grafting fibroblasts genetically modified to produce $\mathrm{L}$ dopa in a rat model of Parkinson disease. Proc Natl Acad Sci USA 1989; 86 : 9011-4.

12. Chasse JF, Levrero M, Kamoun $P$, et al.L'adénovirus: vecteur de thérapie génique? médecine/sciences 1989; $5: 331$-7.

13. Wu CH, Wilson JM, Wu GY. Targeting genes : delivery and persistent expression of a foreign gene driven by mammalian regulatory elements in vivo. J Biol Chem 1989; 264 16985-7. 